\title{
THE C-SHAPED SECOND MANDIBULAR MOLAR AND INTENTIONAL REPLANTATION
}

\author{
Elżbieta Bołtacz-Rzepkowska' ${ }^{1}$ Agnieszka Żęcin², Michał Łęski \\ 'Department of Conservative Dentistry, Medical University of Lodz, Poland \\ 2Private Dental Office, Agnieszka Żęcin TRIDENT PLUS, Lodz, Poland \\ ${ }^{3}$ Department of Endodontics, Medical University of Lodz, Poland
}

\begin{abstract}
The aim of this case report was to describe the endodontic and surgical treatment of a C-shaped mandibular left second molar. The procedure was performed as re-treatment of an initially failed treatment. During re-treatment, the C-shaped canal was prepared chemo-mechanically and filled. Although clinical and radiological evidence of healing was present after one and two years, the lingual wall of the crown was fractured after five years. The hard tissues were restored with post, core, and porcelain-fused-to-metal (PFM) crown. After three years, clinical examination revealed an $8 \mathrm{~mm}$ periodontal pocket in the lingual part of the root, sensation to percussion and tooth mobility. The symptoms suggested root fracture, which required surgical verification. It was decided to perform tooth extraction and intentional replantation if the fracture were not confirmed. The apex was resected and the root end was prepared and filled with mineral trioxide aggregate (MTA). The lingual groove extending from the coronal part of the tooth to the apex was also filled with MTA. The tooth was replanted and splinted with a fiber splint and flow composite for two weeks. The tooth appeared to be clinically asymptomatic after one and two years of the follow-up, and periradicular radiolucency decreased over time. However, signs of ankylosis were observed five years after surgery. In the present case, re-treatment and intentional replantation allowed the tooth to be retained in the oral cavity despite the occurrence of the complication in a form of ankylosis. Sixteen years have passed since the tooth retreatment and 8 years since the procedure of intentional replantation.
\end{abstract}

KEY wORDS: C-shaped canal, intentional replantation, MTA cement.

J Stoma 2018; 71, 3: 308-314

DOI: https://doi.org/10.5114/jos.2018.80645

\section{INTRODUCTION}

The dentist's skills and knowledge of the anatomy of the root canal system are key factors in achieving good results with endodontic treatment. Modern diagnostic techniques allow dentists to treat teeth with atypical anatomy. A C-shaped root canal system is an abnormality in multi-rooted teeth whose canal orifices are fused in the pulp chamber floor, resembling the letter " $\mathrm{C}$ " [1].
According to Manning [2], the failure of Hertwig's epithelial root sheath to fuse on the lingual or buccal root surface is the main cause of $\mathrm{C}$-shaped roots, which always contain a $\mathrm{C}$-shaped canal.

The treatment of C-shaped canals is complicated by two key causes. The first is that correct disinfection and obturation of the atypical morphological configuration is difficult, while the second is associated with a characteristic structure of the root whose cross section is
JOURNAL OF STOMATOLOGY CZASOPISMO STOMATOLOGICZNE
ADDRESS FOR CORRESPONDENCE:

dr hab. n. med. prof. nadzw. Elżbieta Bołtacz-Rzepkowska, Zakład Stomatologii Zachowawczej, 251 Pomorska St., 91-503 Łódź, phone: +42 67574 18, e-mail: elzbieta.boltacz-rzepkowska@umed.lodz.pl 

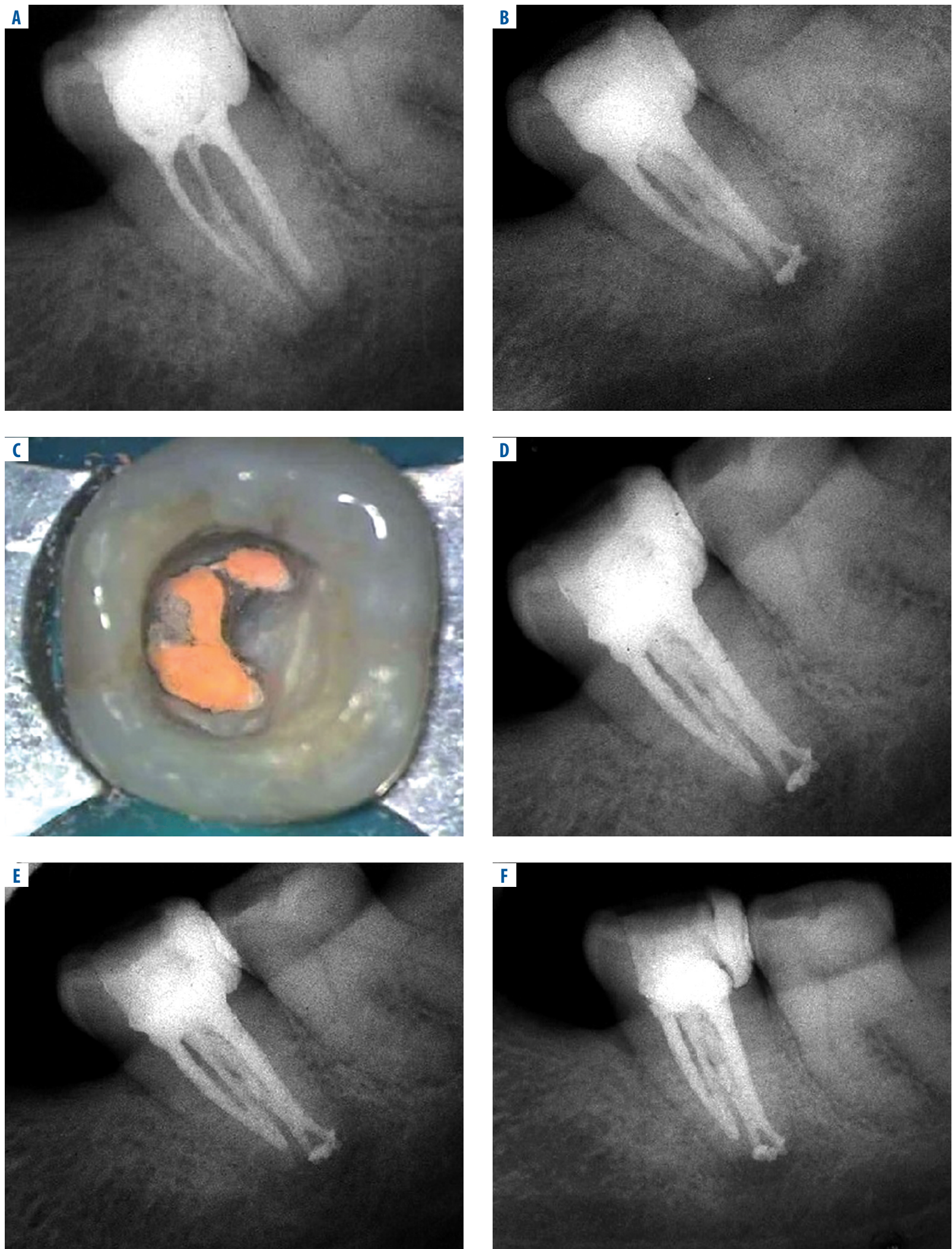

FIGURE 1. A) Periradicular radiolucency. Showing three canal fillings. B) C-shaped canal filling. C) Surgical operating microscope (SOM) view of the pulp chamber floor and the C-shaped canal filling. D) One-year recall examination. E) Two-year recall examination. F) Five-year recall examination. The lingual wall of the crown was fractured subgingivally 
also C-shaped and with the presence of a long groove extending from the coronal part of the tooth to the apex on the lingual or vestibular surface of the root, which was formed by the fusion of roots. Gao et al. [3] stated that the C-shaped canals are frequently eccentric to the groove, resulting in a very thin wall of the root, which can cause perforation or root fracture. Therefore, the treatment of C-shaped teeth is difficult to perform, prognosis is uncertain and early or late complications may occur.

The present study describes a case of endodontic re-treatment and surgical treatment of a mandibular left second molar with a C-shaped root canal system.

\section{CASE REPORT}

In 1999, a 58-year-old man attended the dental office with a toothache of tooth 37 . The spontaneous toothache and tenderness to biting had increased over a period of two days. The patient reported that the tooth had been treated endodontically a few months earlier. Intra-oral examination revealed redness and inflammation of the mucosa in the area of tooth 37 . The tooth was tender to percussion. Periodontal probing revealed no pathological pocket. The radiograph showed periradicular radiolucency and fillings in three canals. The diagnosis was of previously treated symptomatic apical periodontitis (Fig. 1A).

After clinical examination, tooth 37 was re-treated. The old filling material was removed from the root canal. Using a surgical operating microscope (SOM), a round orifice was revealed in the ML area. The second C-shaped orifice was elongated from the MB area to the distal portion. After removing the old filling material, pus was evacuated from the canal. The root canal system was prepared chemomechanically with ProFile (Dentsply Maillefer Ballaigues, Switzerland) rotary instruments, MAF \#40 with taper $4 \%$, using $2.5 \%$ sodium hypochlorite and $17 \%$ EDTA as an irrigant. After preparation, the root canal system was temporarily filled with calcium hydroxide for two weeks, and the round ML canal was filled permanently using the gutta-percha cold lateral compaction technique. The C-shaped canal was filled with gutta-percha and $\mathrm{AH}$ Plus (Dentsply DeTrey, Konstanz, Germany) sealer using the single cone technique in the apical portion and the thermo-plasticized injection technique in the middle and coronal portions (Fig. 1B-C). The cavity was restored with plastic material (composite).

Clinical and radiological evidence of healing was present one and two years after completion of the retreatment (Fig. 1D-E).

However, in 2004, five years after retreatment, the lingual wall of the crown was fractured subgingivally (Fig. 1F). The hard tissues were restored with post and core, and porcelain-fused-to-metal (PFM) crown. No symptoms were present after three years.
In 2007, the patient reported toothache, tenderness to biting and mobility of tooth 37 . Clinical examination revealed redness of the alveolar mucosa and an $8-\mathrm{mm}$ periodontal pocket in the lingual part of the root as well as tenderness to percussion (Fig. 2A). The radiograph showed increased peri-radicular radiolucency and thickening of the periodontium (Fig. 2B). The earlier fracture of the lingual wall and presence of a periodontal pocket indicated the possibility of vertical root fracture. Thus it was decided to extract the tooth. In the case of the absence of any root fracture, an intentional replantation would be taken into consideration.

After applying local anesthesia (Citocartin 100-Molteni Dental, Scandicci, Florence, Italy) the tooth was extracted. The root was inspected by endodontic microscope. A groove was found on the lingual part of the tooth root, which extended from the coronal part of the tooth to the apex (Fig. 2C). As no cracks or fractures were found on the surface of the root, it was decided to perform an intentional replantation. After root-end resection, the apical part of the root was prepared using an inverted cone-shaped bur mounted on the low speed handpiece (Fig. 2D). Saline solution was used as the cooling irrigant. The root-end cavity was filled with White Pro Root mineral trioxide aggregate (MTA) (Dentsply, Tulsa Dental, USA) (Fig. 2E). The lingual groove was also prepared using the same bur and filled with White Pro Root MTA (Dentsply, Tulsa Dental, USA) (Fig. 2F). During the procedure, the tooth was kept moist with sterile gauze. The tooth was outside the socket for 15 minutes: the total procedure from tooth extraction to tooth replantation took 25 minutes. After irrigating the socket with saline solution, the tooth was reimplanted and splinted with a fiber splint and flow composite for two weeks (Fig. 3A-D).

After the reimplantation, recall examinations at one and two years revealed periradicular radiolucency (Fig. 4A-B), but it decreased over time. Five years after surgery, signs of ankylosis were observed on a radiograph (Fig. 4C). The clinical examination revealed loss of physiological mobility of the tooth. It was not possible to hear the metallic sound after tapping the tooth, characteristic of ankylosis, as the tooth had been provided with a PFM crown after the fracture. After six years, clinical and radiological examination confirmed the symptoms of ankylosis (Fig. 4D).

\section{DISCUSSION}

Cook and Cox first described the C-shaped canal system in 1979 [6]. The anatomy of the C-shaped canal is difficult to diagnose on the basis of a pretreatment radiograph. In 2001, Lambrianidis [7] described the radiological criteria for diagnosing C-shaped canals as a conical root shape with almost invisible canals and the pulp chamber floor. 

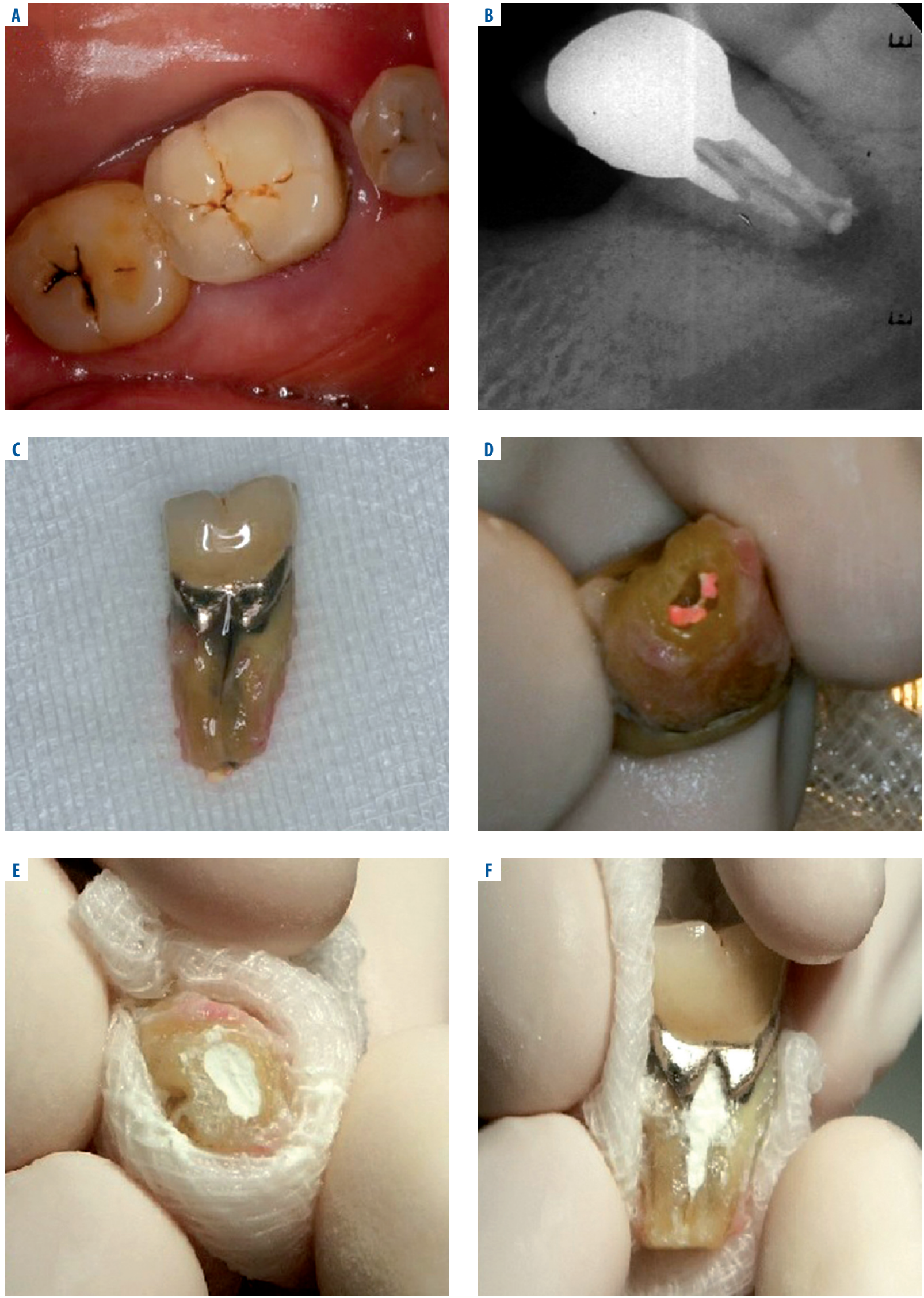

FIGURE 2. A) Redness of the mucosa indicates a periodontal pocket. B) Periradicular radiolucency and thickening of the periodontium. C) Lingual groove. D) Photograph after apicoectomy and root-end preparation. E) Root-end filling with ProRoot mineral trioxide aggregate (MTA). F) Lingual groove filling with ProRoot MTA 

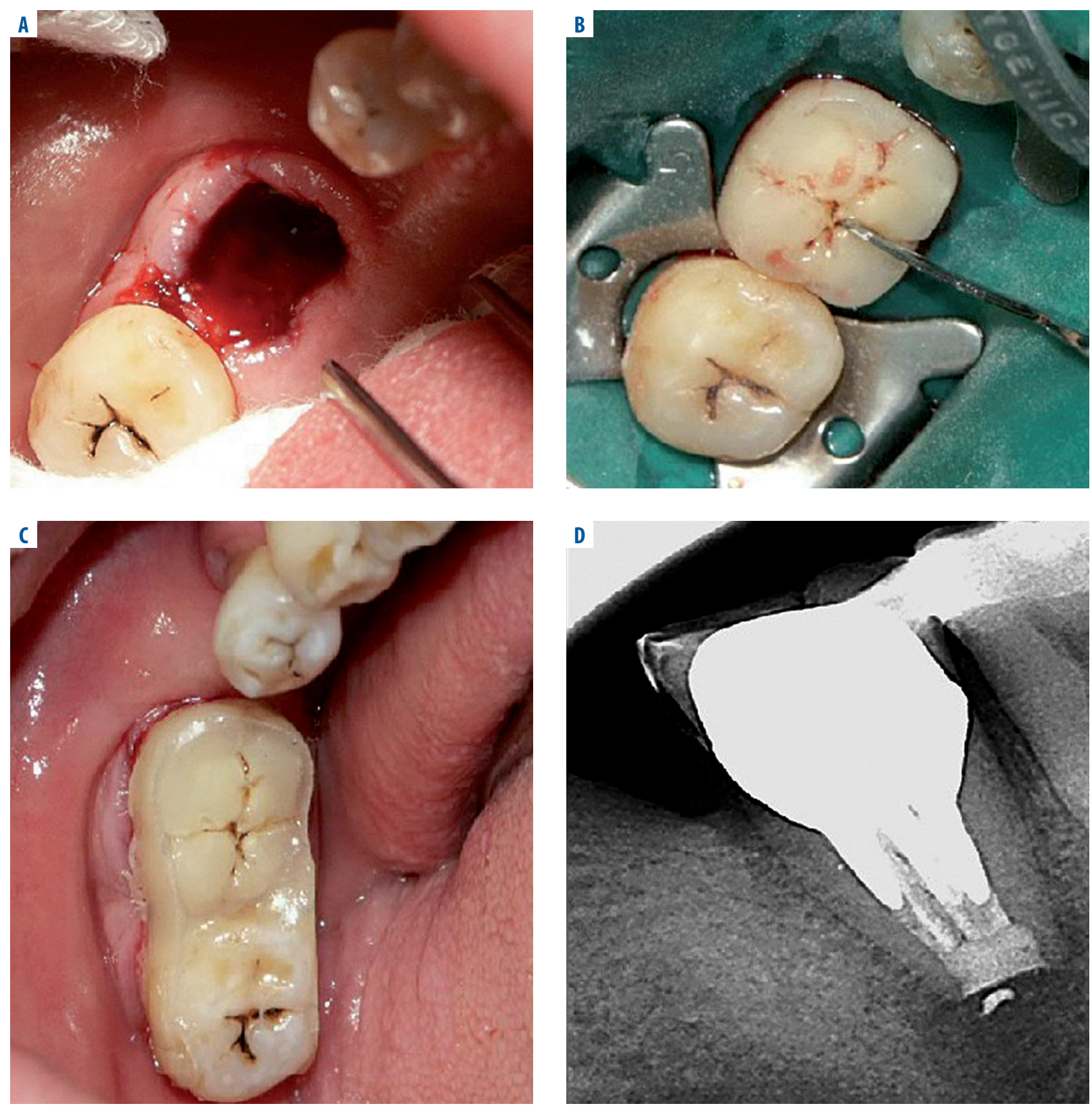

FIGURE 3. A) Socket after tooth extraction filled with blood. B) Preparation for splinting. C) Splinted teeth. D) X-ray of replanted tooth after splinting

The following classification of C-shaped canals based on root cross-sections was proposed in 1991 by Melton etal.: category $\mathrm{I}(\mathrm{C} 1)$, a continuous C-shaped canal running from the pulp chamber to the apex defining a C-shaped outline without any separation; category II (C2), a semicolon-shaped; orifice in which dentin separates a main C-shaped canal from one mesial distinct canal; category III (C3), two or more separate canals (1). A C-shaped canal (C1) with only one canal is very rare. The C-shaped root canal system is usually irregular with lateral canals and anastomoses [3-5, 8, 9].

In the present case, the canal system was classified as C2 with two separate canals: a round ML canal and a semicolon-shaped $\mathrm{MB}+\mathrm{D}$ canal that merged together. Irregular areas in the C-shaped canal system contain soft-tissue remnants or infected debris that may escape thorough cleaning or filling procedures [10]. Our case is an example of the difficulties faced in treating C-shaped canals. The failure of the first treatment was due to poor identification of the morphological structure of the treated tooth. The canals were treated as three separate canals without thorough debridement of the C-shaped canal system, which was the cause of endodontic complications. The radiograph taken 6 months after the primary treatment revealed periradicular radiolucency around the apex. 

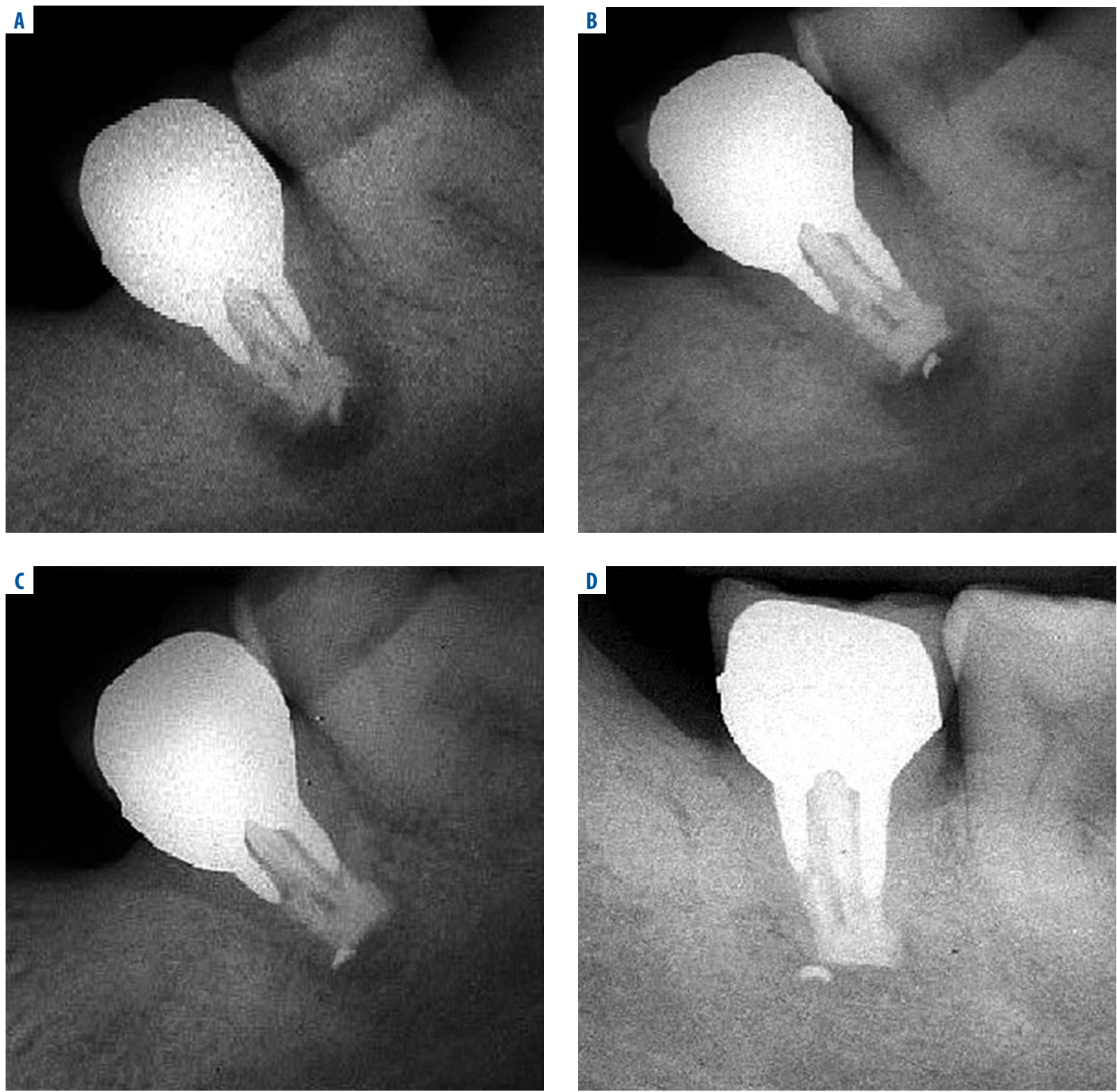

FIGURE 4. A) One-year recall examination after intentional replantation. B) Two-year recall examination after intentional replantation. C) Three-year recall examination after intentional replantation - ankylosis. D) Six-year recall examination after intentional replantation - ankylosis

After re-treatment, complete healing of the lesions was not observed. Several intra- and extra-canal factors can affect the healing of lesions in the periradicular tissues after endodontic retreatment. Bacteria remaining in the unprepared root canal system space constitute intra-canal factors. Extra-canal factors such as the presence of a filling material beyond the root apex or an extra-canal biofilm may prolong healing. An impairment of the balance between the protective ability of the body and the virulence of the pathogen is also probable [11].

In each case of endodontic treatment, a tight seal and durability of crown reconstruction are important. It protects the tooth from re-infection as well as from fracture [12]. Improper crown reconstruction of the treated tooth, which did not encompass the entire occlusal surface, caused the fracture of the lingual wall and the disruption of the circular ligament.

In our case, a deep pocket was observed on the lingual side of the tooth, indicating the presence of a vertical root fracture, which required surgical verification. The replantation was intended due to the suspicion that the source of infection was located in the root canal system and in the groove on the lingual surface of the root. This suspicion was justified by the fact that the peri-radicular lesion was not completely healed after retreatment of the tooth. The deep pocket resulted from the exposure and infection of the groove after the crown fracture which allowed pathogens to infect the peri- 
radicular tissues, maintain the inflammation, and induce the development of endo-perio lesions. As root apex resection with root-end filling and curettage was contraindicated due to the position of the pathological pocket (from the lingual part of the root) and the position of the second mandibular molar, intentional replantation was performed. All these aspects required surgical verification. The presence of fused roots provided good conditions for such a surgical procedure in a predictable way. During the replantation, vertical fracture was excluded, but unsound canal filling in the apical area and the lingual groove were observed. These outcomes confirmed the correctness of our management.

There are different opinions on the management of the alveolus in the intentional replantation. Previously, clinicians advocated curettage of the socket after tooth removal. It is now known that the walls of the socket should not be touched and that the apical region should only be gently aspirated if needed and the socket should be irrigated with saline solution [13, 14]. Moiseiwitsch [15] reported little difference in success with or without apical curettage. In the present case, the socket was only irrigated with saline solution.

Intentional replantation is a surgical procedure in which the tooth is extracted and treated extra-orally to restore the tooth or correct an endodontic failure and then re-inserted in its socket. This procedure has a risk of root resorption and ankylosis due to the extended extra-oral time that could affect the viability of the periodontal cells covering the root surface [16-19]. In our case, the surgical procedure time was $25 \mathrm{~min}$ utes including the extraction. The out-of-socket time including root-end preparation and filling, as well as the preparation and filling of the groove extending from the coronal part of the tooth to the apex on the lingual surface of the root, was 15 minutes.

\section{CONCLUSIONS}

The present case report describes the retreatment and intentional replantation of a second mandibular. Despite the observed radiological symptoms of ankylosis, our management allowed the tooth to be functional for sixteen years after retreatment and eight years after intentional replantation.

\section{CONFLICT OF INTEREST}

The authors declare no potential conflicts of interest with respect to the research, authorship, and/or publication of this article.

\section{References}

1. Melton DC, Krell KV, Fullrer MW. Anatomical and histological features of C-shaped canals. J Endod 1991; 17: 384-388.
2. Manning SA. Root canal anatomy of mandibular second molars. Part II. C-shaped canals. Int Endod J 1990; 23: 40-45.

3. Gao Y, Fan B, Cheung GSP, et al. C-shaped canal system in mandibular second molars part IV: 3-D morphological analysis and transverse. J Endod 2006; 32: 1062-1065.

4. Pilo R, Corcino G, Tamse A. Residual dentin thickness in mandibular premolars prepared with hand and rotatory instruments. J Endod 1998; 24: 401-404.

5. Fan B, Min Y, Lu G, et al. Negotiation of C-shaped canal systems in mandibular second molars. J Endod 2009; 35: 1003-1008.

6. Cooke HG, Cox FL. C-shaped cnal configutation in mandibulars molars. J Am Dent Assoc 1979; 99: 836-839.

7. Lmabrinidis T, Lyroudia K, Pandeidou O, Nicolaou A. Evaluation of periapical radiographs in the recognition of $\mathrm{C}$-shaped mandibular second molars. Int Endod J 2001; 34: 458-462.

8. Fan B, Cheung GSP, Fan M, et al. C-shaped canal system in mandibuar second molars: part I-anatomical features. J Endod 2004; 30: 899-903.

9. Żęcin A, Bołtacz-Rzepkowska E. Root canal treatment of C-shaped canals. J Stoma 2005; 58: 302-309.

10. Weine FS. The C-shaped mandibular second molar: incidence and other considerations. Members of the Arizona Endodontic Association. J Endod 1998; 24: 372-375.

11. Siqueira JF Jr. Aetiology of root canal treatment failure: why well-treated teeth can fail. Int Endod J 2001; 34: 1-10.

12. Zarow M, Devoto W, Saracinelli M. Reconstruction of endodontically treated posterior teeth - with or without post? Guidelines for the dental practitioner. Eur J Esthet Dent 2009; 4: 312-327.

13. Kratchman S. Tooth replantation. In: Kim S, Pecora G, Rubinstein A. Color atlas of microsurgery in endodontics. W.B. Saunders Co., Philadelphia 2001; 131.

14. Kratchman S. Intentional replantation. Dent Clin North Am 1997; 41: 603-617.

15. Moiseiwitsch J. Intentional replantation and transplantation: I.B. Bender, DDS. J Endod 1992; 18: 415-418.

16. Bender IB, Rossman LE. Intentional replantation of endodontically treated teeth. Oral Surg Oral Med Oral Pathol 1993; 76: 623-630.

17. Andreasen JO, Borum MK, Jacobsen HL, Andreasen FM. Replantation of 400 avulsed permanent incisors. Part 1. Diagnosis of healing complications. Endod Dent Traumatol 1995; 11: 51-58.

18. Peer M. Intentional replantation - a "last resort" treatment or a conventional treatment procedure? Nine case reports. Dent Traumatol 2004; 20: 48-55.

19. Filippi A, Pohl Y, von Arx T. Treatment of replacement resorption by intentional replantation, resection of the ankylosed sites, and Emdogain - results of a 6-year survey. Dent Traumatol 2006; 22: 307-311. 